http://jmscr.igmpublication.org/home/ ISSN (e)-2347-176x ISSN (p) 2455-0450

crossref DOI: https://dx.doi.org/10.18535/jmscr/v7i11.13

Journal Of Medical Science And Clinical Research

Original Research Article

\title{
Study of Role of Vitamin D3 Supplementation in Patients Suffering from Dry Eye Syndrome (DES) Attending in Tertiary Care Hospital, at S.K.M.C.H. Muzaffarpur, Bihar
}

\author{
Authors \\ Dr Ramakant Thakur ${ }^{1}$, Dr Rajiv Kumar Singh ${ }^{2 *}$ \\ ${ }^{1}$ Assistant Professor, Department of Ophthalmology, S. K. Medical College, and Hospital, Muzaffarpur \\ ${ }^{2}$ Associate Professor and H.O.D., Department of Ophthalmology, S. K. Medical College, and Hospital \\ Muzaffarpur \\ *Corresponding Author \\ Dr Rajiv Kumar Singh
}

Associate Professor and H.O.D, Department of Ophthalmology, S. K. Medical College, and Hospital,

Muzaffarpur, India

\begin{abstract}
Objective: Present study was conducted to evaluate the role and effect of Vitamin D supplementation on tear film and in ameliorating the symptoms of Dry Eye Syndrome (DES).

Materials and Methods: A total of 56 patients aged between 38 to 75 years presenting with symptoms related to dry eye Syndrome attending in eye OPD were included in the study. Vitamin D estimation was done before starting treatment, at 2 weeks, 4 weeks and 8 weeks intervals after Vitamin $D$ supplementation. Quantitative determination of Vitamin D estimation was done by fully automated Architect machine supplied by Roche pharma by CMIA (Chemiluminiscent Microparticle Immunoassay) methods. Schirmer's test and Tear break up time (TBUT) were done to assessment of the tear film. Eye discomfort was assessed by Ocular surface disease index (OSDI).

Results: Out of 56 patients mean age of the patients were $47.5 \pm 22.5$ years. Mean serum $25 \mathrm{OH}-\mathrm{D}$ level was found to be $l 1.4 \pm 3.6 \mathrm{ng} / \mathrm{ml}$. Mean Tear break up time (TBUT) was $2.6 \pm 2.2 \mathrm{~s}$ at pretreatment. It increased to $4.2 \pm 1.4 \mathrm{~s}$ after 2 weeks and to $5.2 \pm 1.6 \mathrm{~s}$ after 4 weeks and to $5.9 \pm 1.4 \mathrm{~s}$ after 8 weeks. Schirmer's test at pretreatment level was $5.7 \pm 2.2 \mathrm{~mm}, 7.8 \pm 4.3 \mathrm{~mm}$ after 2 weeks, $8.3 \pm 5.6 \mathrm{~mm}$ after 4 weeks and $9.9 \pm 6.3 \mathrm{~mm}$ after 8 weeks. Ocular surface disease index (OSDI) was $29.56 \pm 21.2$ at pretreatment, $24.9 \pm 20.5$ after 2 weeks, $21.9 \pm 19.13$ after 4 weeks and $19.52 \pm 17.3$ after 8 weeks.

Conclusion: Tear secretion is a major factor in the development of DES. Reduced tear production results in tear instability and ocular surface damage. Vitamin D supplementation was shown to promote tear secretion, reduce tear instability, and reduce inflammation of the ocular surface and eyelid margin

Keywords: Dry Eye Syndrome (DES), vitamin D supplementation, Tear break up time (TBUT), Ocular surface disease index (OSDI).
\end{abstract}

\section{Introduction}

Dry Eye Syndrome (DES) is a common ocular disease of decreased tears and ocular surface disturbance leading to tear film instability, resulting in ocular discomfort fatigue and pain with diminution of vision. The disease usually is 
not curable, much to the frustration of the patient and treating ophthalmologist. Recently, Vitamin D deficiency has been suggested to be a contributory factor in DES.

Treatment for DES includes the use of artificial tears, anti-inflammatory drugs, autologous serum, and punctal occlusion. Artificial tears contain carboxymethyl cellulose or hyaluronate sodium and act as a lubricating agent at the ocular surface. Treatment with artificial tears only gives palliative relief of eye irritation in patients with aqueous tear deficiency, but do not treat the underlying inflammation or reverse conjunctival squamous metaplasia in chronic DES. Conventional treatments using topical drugs and punctal occlusion are not effective in the treatment of chronic ocular pain in some patients with DES.

Recently, vitamin D deficiency has been suggested to be a contributory factor in DES. An association between DES and serum 25hydroxyvitamin D $(25(\mathrm{OH}) \mathrm{D})$ concentration has been suggested. It has been reported that vitamin D plays an immuno-modulatory role in innate and adaptive immunity. Vitamin D and the vitamin D receptor (VDR) regulate several genes involved in inflammation, immunity, cellular proliferation, differentiation, and apoptosis. However, the effect of vitamin D supplementation on DES has not been reported.

Vitamin D acts an immunomodulatory effect so present study was conducted to evaluate the role and effect of Vitamin D supplementation on tear film and in ameliorating the symptoms of DES that was refractory to conventional treatment.

\section{Materials and Methods}

Present study was conducted in the Department of Ophthalmology, Sri Krishna Medical College and Hospital, Muzaffarpur, Bihar during the period of May 2019 to September 2019.A total of 56 patients aged between 38 to 75 years presenting with symptoms related to dry eye attending in eye OPD, who were refractory to artificial tear supplements and having Vitamin D deficiency, as shown by serum 25 OH-D concentrations were selected for the study. Patients with Sjogren's syndrome, corneal grafting, corneal opacity and keratoconus were excluded from the study. Vitamin D estimation was done before starting treatment, at 2 weeks, 4 weeks and 8 weeks intervals after Vitamin D supplementation. After thorough clinical history, all the patients were subjected to proper clinical examination and referred to biochemistry department for Vitamin D estimation. Quantitative determination of Vitamin D estimation was done by fully automated Architect machine supplied by Roche pharma by CMIA (Chemiluminiscent Microparticle Immunoassay) methods. Schirmer's test and Tear break up time (TBUT) were done to assessment of the tear film. Eye discomfort was assessed by ocular surface disease index (OSDI).

\section{Results}

Out of 56 patients mean age of the patients were $47.5 \pm 22.5$ years. Mean serum 25 OH-D level was found to be $11.4 \pm 3.6 \mathrm{ng} / \mathrm{ml}$.

Mean Tear break up time (TBUT) was $2.6 \pm 2.2 \mathrm{~s}$ at pretreatment. It increased to $4.2 \pm 1.4 \mathrm{~s}$ after 2 weeks and to $5.2 \pm 1.6 \mathrm{~s}$ after 4 weeks and to $5.9 \pm$ $1.4 \mathrm{~s}$ after 8 weeks.

Schirmer's test at pretreatment level was $5.7 \pm$ $2.2 \mathrm{~mm}, 7.8 \pm 4.3 \mathrm{~mm}$ after 2 weeks, $8.3 \pm 5.6 \mathrm{~mm}$ after 4 weeks and $9.9 \pm 6.3 \mathrm{~mm}$ after 8 weeks.

Ocular surface disease index (OSDI) was $29.56 \pm$ 21.2 at pretreatment, $24.9 \pm 20.5$ after 2 weeks, $21.9 \pm 19.13$ after 4 weeks and $19.52 \pm 17.3$ after 8 weeks.

\section{Discussion}

Dry eye syndrome (DES) is a common ocular disease that is characterized by tear instability, ocular surface inflammation, and irritable eye symptoms like chronic ocular fatigue and pain. The reduction of tear secretion from lacrimal glands leads to aqueous deficiency DES and eyelid margin inflammation and meibomian gland dysfunction (MGD) have been suggested as major causes of evaporative type of DES. DES has been shown to cause inflammation of the ocular surface 
that is evidenced by increased levels of inflammatory cytokines in the tear fluid and corneal and conjunctival epithelia, and infiltration of $\mathrm{CD}^{+} \mathrm{T}$ cells into the conjunctiva.

The nuclear factor- $\kappa \mathrm{B}(\mathrm{NF}-\kappa \mathrm{B})$ and mitogenactivated protein kinase (MAPK) pathways are activated in DES. NF- $\kappa \mathrm{B}$ is stimulated by proinflammatory cytokines such as interleukin 1 (IL-1) and tumor necrosis factor $\alpha(\mathrm{TNF} \alpha)$. NF- $\mathrm{kB}$ regulates the expression of a wide variety of proinflammatory genes, including the genes for cytokines, chemokines, and adhesion molecules. $\mathrm{NF}-\kappa \mathrm{B}$ plays a critical role in ocular surface inflammation and disease.

T-helper 17 cells play an important role in DES. Th-17 cytokines are associated with disruption in corneal epithelial barrier function and can induce IL-6, transforming growth factor- $\beta$ (TGF- $\beta$ ), IL23 and IL-17A on the ocular surface of DES patients. These pathogeneses contribute to the development of the ocular irritation associated with DES. It has been suggested that the impact of severe DES on quality of life is similar to the impact of moderate to severe angina.

The aim of this study was to find the effect of Vitamin D3 supplementation in DES not responding to usual treatment. Serum 25 (OH-D) concentrations was used as an indicator of Vitamin D status of the person. Cholecalciferol oral spray 3000 i.u.per day was used for the treatment of Vitamin D deficiency. This study showed that Vitamin D supplementation improved tear break up time (TBUT) and tear secretion as shown by Schirmer's test. Ocular discomfort and pain lessened with reduced tear instability and ocular surface damage.

DES is an inflammatory disease resulting from activation of innate inflammatory pathways in resident ocular surface cells. 25 (OH-D) ameliorates the ocular surface inflammation by down regulating Th 17 cytokines,IL-1,IL-2 and IFN-Y concentration in tears, which is significantly increased in DES patients with the concentration being parallel to the severity of disease. Cholecalciferol oral spray 3000 IU should be supplemented daily for management of refractory cases of DES.

\section{Conclusion}

Vitamin D3supplementation improved the symptoms of DES as shown by increased tear secretion and TBUT with reduced ocular surface inflammation and hyperemia. So, it seems to be a useful treatment for DES patients, refractory to conventional treatment.

\section{References}

1. Stevenson, W. Chauhan, S.K. \& Dana, R. Dry eye disease: An immune related ocular surface disorder. Arch Opothalmol 130, 90-100 (2012)

2. Tan, X. et al. Analysis of Th 17 associated cytokines in tears of patients with Dry Eye Syndrome. Eye (Land) 28, 608-13, 2014.

3. Galor, A et al. Effect of a Mediterranean dietary pattern and Vit. D levels on Dry Eye Syndrome. Cornea 33, 437- 441 (2014)

4. Albert DM Jakobiec FA Water and electrolyte secretion and fluid modification. Principles and Practice of Ophthalmology Basic Sciences (Vol 1.1A.)Philadelphia, $\mathrm{Pa}$ WB Saunders Co1995;sect 1B, chap 29B.Google Scholar

5. Miljanovic B., Dana R., Sullivan D. A. \& Schaumberg D. A. Impact of dry eye syndrome on vision-related quality of life. Am J Ophthalmol 143, 409-415 (2007).

6. The definition and classification of dry eye disease: report of the definition and classification subcommittee of the International Dry Eye WorkShop (2007). Ocul Surf 5, 75-92 (2007).

7. Zheng $X$. et al. Desiccating stress promotion of Th17 differentiation by ocular surface tissues through a dendritic cell-mediated pathway. Invest Ophthalmol Vis Sci 51, 3083-3091 (2010). 
8. Pflugfelder S. C., de Paiva C. S., Li D. Q. \& Stern M. E. Epithelial-immune cell interaction in dry eye. Cornea 27 Suppl 1, S9-S11 (2008).

9. Lan $W$. et al. Nuclear Factor- $\kappa B$ : central regulator in ocular surface inflammation and diseases. Ocul Surf 10, 137-148 (2012).

10. Lawrence $T$. The nuclear factor $N F-\kappa B$ pathway in inflammation. Cold Spring Harb Perspect Biol 1, a001651 (2009).

11. Abu-Amer Y. et al. Tumor necrosis factoralpha activation of nuclear transcription factor-kappaB in marrow macrophages is mediated by c-Src tyrosine phosphorylation of Ikappa Balpha. J BiolChem 273, 29417-29423 (1998).

12. Luo L. et al. Experimental dry eye stimulates production of inflammatory cytokines and MMP-9 and activates MAPK signaling pathways on the ocular surface. Invest Ophthalmol Vis Sci 45, 4293-4301 (2004). 